\title{
TECNOLOGÍA DE INFORMACIÓN Y COMUNICACIÓN EN LA MEJORA DE LA EXPERIENCIA DE LOS VIAJEROS DE LA GENERACIÓN MILLENNIALS
}

INFORMATION AND COMMUNICATION TECHNOLOGY TO ENHANCE THE MILLENNIAL GENERATION TRAVELLER EXPERIENCE

\author{
Ana María Miranda Zavala \\ Doctora en Ciencias Administrativas y Negocios Internacionales. \\ Universidad Autónoma de Baja California. Tijuana, (México). \\ E-mail: amiranda@uabc.edu.mx ORCID: https://orcid.org/0000-0001-6796-2309 \\ Isaac Gruz Estrada \\ Doctor en Planeación Estratégica. \\ Universidad Autónoma de Baja California. Tijuana, (México). \\ E-mail: icruz@uabc.edu.mx ORCID: https://orcid.org/0000-0002-7673-0195
}

Recepción: 13/10/2020 Aceptación: 30/11/2020 Publicación: 29/12/2020

\section{Citación sugerida:}

Miranda, A. M., y Cruz, I. (2020). Tecnología de Información y Comunicación en la mejora de la experiencia de los viajeros de la generación millennials. 3C TIC. Cuadernos de desarrollo aplicados a las TIC, 9(4), 67-87. https://doi. org/10.17993/3ctic.2020.94.67-87 


\section{RESUMEN}

El objetivo versa en analizar la experiencia del viajero de la generación millennials en el uso de las tecnologías durante la estancia en los destinos turísticos. Metodología, con base 95\% de confianza y 5\% de error admitido, se aplicó un cuestionario a 385 millennials con edad entre 20 y 39 años, residentes de la ciudad de Tijuana, Baja California, México, que realizaron al menos cinco viajes fuera del Estado en 2019. Se realizó análisis descriptivo de los datos y regresión lineal, para identificar la relación de servicios tecnológicos y apps de Internet empleadas durante el viaje con la experiencia obtenida por los millennials. Resultados, los servicios tecnológicos que utilizan con más frecuencia durante su visita al destino, es la geolocalización que optimiza los tiempos del recorrido en los lugares de interés. Asimismo, comparte sus experiencias y realizan recomendaciones mediante Facebook, WhatsApp, Instagram y Google. Se obtuvo una correlación media positiva entre las variables analizadas: frecuencia de uso de los servicios tecnológicos y empleo de las apps con la experiencia del viaje en los millennials. Se concluye, el uso de los servicios y apps tecnológicas, están asociados en un 34,5\% con la experiencia positiva de los viajeros millennials en los destinos que visitan.

\section{PALABRAS CLAVE}

Millennials, Comunicación, Tecnología, Viajeros, TIC, Internet, Turismo, Redes Sociales, Experiencia. 


\section{ABSTRACT}

The objective is to analyze the experience of the traveler of the millennial generation in the use of technologies while staying in tourist destinations. Methodology, based on 95\% confidence and 5\% admitted error, a questionnaire was applied to 385 millennials aged between 20 and 39 years, residents of the city of Tijuana, Baja California, Mexico, who made at least five trips outside the Status in 2019. A descriptive analysis of the data and linear regression were carried out to identify the relationship of technological services and Internet apps used during the trip with the experience obtained by millennials. Results, the technological services that they use most frequently during their visit to the destination, is the geolocation that optimizes the travel times in the places of interest. Likewise, they share their experiences and make recommendations through Facebook, WhatsApp, Instagram and Google. A positive mean correlation was obtained between the variables analyzed: frequency of use of technological services and use of apps with the travel experience in millennials. It is concluded that the use of technological services and apps is associated in 34,5\% with the positive experience of millennial travelers in the destinations they visit.

\section{KEYWORDS}

Millennials, Communication, Technology, Travellers, ICT, Internet, Tourism, Social networks, Experience. 


\section{INTRODUCCIÓN}

La revolución de las Tecnologías de Información y Comunicación (TIC) y su impacto en niños y jóvenes, ha provocado la necesidad de acceso a grandes volúmenes de información disponible en la web (Caballero y Baigorri, 2013). Además, con el aumento exponencial del uso de las herramientas y plataformas tecnológicas, aparecen nuevos nichos de mercados, los cuales se han vuelto consumidores y productores de contenido a la vez, este rol ha impulsado el trabajo colaborativo en la red, más allá de los intereses personales (Feixa, Fernández-Planells, y Figueras-Maz, 2016). Por otra parte, el teléfono móvil inteligente, se ha convertido en un elemento indispensable y una extensión necesaria en el funcionamiento de la vida de las personas (Díaz-Sarmiento, López-Lambraño, y Roncallo-Lafont, 2017).

En este caso, se analiza la generación Y, también conocida como millennials son personas nacidas entre 1981 hasta el año 2000, crecieron en una época de grandes avances tecnológicos, que ha conducido a una relación intensiva y fascinación por utilizar los medios digitales para disponer de los servicios de las empresas (Carvalho dos Santos et al., 2015; Díaz-Sarmiento et al., 2017). La población que conforma este segmento se constituye sustancialmente a partir de la expansión de Internet y globalización de los mercados, se pronuncia con tener una conectividad a escala mundial, con la necesidad de acceso e inmediatez a la información (Rossi et al., 2018). Los millennials buscan formas peculiares de entretenimiento, con propósitos gratificantes en las experiencias que desean obtener, en su mayoría planifican sus viajes usando recursos tecnológicos, lo que también contempla diferentes hábitos de compra en comparación a las generaciones que los anteceden (Ordaz-Mejía y Osorio-García, 2016). Los jóvenes millennials usan las diversas aplicaciones disponibles en la web como sus principales medios de información y comunicación con las organizaciones turísticas, aprovechan la disponibilidad de la tecnología para la compra de los servicios disponibles en el destino, además, se distinguen por personalizar su desplazamiento para optimizar el tiempo y hacer más eficiente su travesía (Castro, Peñaloza y Tamayo, 2018).

Con este escenario, la innovación tecnológica se ha vuelto una necesidad en la industria del Turismo, tomando en cuenta las oportunidades que representan, para conectar a las personas con los atractivos 
naturales y culturales de cada región del mundo (López-Rodríguez y López, 2018). Es indispensable encontrar los medios que permita a los diferentes segmentos de la población, reconocer, sensibilizar y disfrutar estar vinculados con los servicios y atractivos de cada comunidad. El objetivo de la investigación versa en analizar la relación que existe, entre el uso de los servicios tecnológicos empleados para los destinos visitados, así como la frecuencia de uso de las apps del dispositivo móvil, con la experiencia de viaje obtenida por los turistas de la generación millennials. Los participantes de la investigación confirmaron realizar al menos cinco viajes fuera del Estado de Baja California en el año 2019, la población de estudio manejó el teléfono inteligente o Smartphone para comunicarse, obtener información y comprar servicios requeridos para el desarrollo de sus travesías.

\subsection{TIC EN LA INDUSTRIA DEL TURISMO.}

El avance tecnológico y su penetración en todos los aspectos de la vida humana, la industria turística ha aplicado una variedad de tecnologías para facilitar las actividades de viaje para mejorar las experiencias de destino de los viajeros (Zhang et al., 2019). En cuanto a las tecnologías móviles se han convertido en un elemento clave, que beneficia la experiencia y satisfacción de los turistas (Ballesteros, Tavera, y Castaño, 2014; Chang y Wei-Cheng, 2018). El desarrollo de la tecnología móvil desempeña un papel cada vez más importante en la experiencia turística, facilitando la disposición de los servicios en el destino de interés (Organización Mundial del Turismo, 2015; Secretaría de Turismo (SEGTUR), 2018; Dickinson, Hibbert y Filimonau, 2016), estos dispositivos tiene una utilidad considerable en el dominio del turismo, debido a que los viajeros se encuentran en lugares desconocidos y buscan información para resolver problemas prácticos de viaje y enriquecer la experiencia turística (Dickinson et al., 2016). A través, de estos dispositivos es posible organizar los viajes, comparar destinos de interés, entre más funciones que pueden aprovecharse al utilizar esta tecnología (Chang y Wei-Cheng, 2018). Invertir en tecnologías interactivas representa una oportunidad para hacer que las visitas sean más accesibles, desarrollar el entretenimiento educativo y, en general, mejora la experiencia del visitante (Ponsignon y Derbaix, 2020). 
El Turismo emplea las TIC, como uno de los componentes indispensables para alcanzar sus objetivos, la tecnología no soluciona todas las necesidades, sin embargo, proporciona recursos prácticos para la comunicación y acceso a la información para todas las personas (Rodríguez-Moreno, 2018). Dentro de este sector, es posible encontrar en la actualidad diversos sitios en Internet con todo tipo de noticias, aplicaciones y recomendaciones acerca las ciudades o comunidades que se desea visitar (Posso y Cardona, 2016). Además, existen numerosos blogs con miles de seguidores, en donde se presentan opiniones individuales, consejos para hacer una maleta con los accesorios indispensables, comentarios de experiencias de lugares donde se logra comer bien y son económicos en destinos dentro del país y en el extranjero (Castro et al., 2018). Las TIC son parte de los cambios en las relaciones, generan mayores incentivos en la cooperación entre competidores, las pequeñas empresas se agrupan en la actualidad para el desarrollo de una página web en común, ofreciendo una misma plataforma en la que se comparten los gastos de operación, esto ocasiona una mayor oportunidad de ser visitados, además de incorporar intermediarios para facilitar la comercialización y distribución de los productos y servicios ofertados (Rodríguez-Moreno, 2018).

Las tecnologías han facilitado el acceso a información turística para los consumidores (Vieira et al., 2017). Los consumidores actuales de este sector requieren encontrar información fiable, con acceso sencillo y flexible para obtener lo que desean, por este motivo resulta fundamental mantener actualizado y en congruencia, lo publicado tanto en el sitio web principal y las redes sociales empleadas por la compañía para compartir el mensaje publicitario y de promoción (Melián y Bulchand, 2015). La tecnología móvil acompaña al viajero en todo momento, brindándole información en tiempo real, facilidad de movimiento, esta herramienta cambia la experiencia de los viajeros, aún lejos de su lugar de origen, se sienten conectados, compartiendo sus experiencias en las redes sociales (Ricaurte-Quijano, Arellano y Naranjo, 2017).

Las tecnologías empleadas de manera adecuada brindan oportunidades a los gobiernos para la resiliencia de los espacios a partir de los elementos que contribuyan al crecimiento económico, al mismo tiempo 
mitigar y reparar las intervenciones que rompen el equilibrio con el entorno, en consecuencia, mejorar la calidad de vida de sus poblaciones y proporcionar mejores condiciones para los visitantes (Linares \& Vásquez, 2018).

\subsection{TURISTAS MILLENNIALS}

Se conoce muy poco del comportamiento turístico de la generación Y, esencialmente qué valores percibidos influyen en su lealtad de destino (Luo et al., 2018). Por lo que, resulta ser complejo predecir el comportamiento de los millennials, su patrón de decisión puede cambiar por algún suceso de última hora, comentario o punto de vista de alguna persona a la que siguen en Internet, la atención al cliente y creación de valor tiene mucho significado para este mercado (Posso y Cardona, 2016; Luo et al., 2018). Entre las causas de su comportamiento por priorizar la búsqueda de información y compra on-line, se debe a que la generación de los millennials prefiere la inmediatez que encuentra mediante Internet, antes de pasar muchas horas en las tiendas físicas para seleccionar y decidir su compra (Simanjuntak, 2019; Shamala, 2013). Además, este segmento de la población permanece mucho tiempo en las redes sociales para intercambiar experiencias, se les identifica por tener un alto nivel de extraversión, que se relaciona con la necesidad de apertura a relacionarse con otros usuarios, con los cuales intercambian opiniones sobre temas de actualidad que están presentes en los diferentes portales de la web (Alan y Kabadayı, 2016). Esta generación comprende mejor cómo conectarse y cómo usar dispositivos digitales, ya que los usan desde su niñez (Djamasbi, Siegel, y Tullis, 2010).

Los millennials son testigos del desarrollo de las TIC y del procesamiento de grandes volúmenes de información en Internet, se caracterizan por ser seguros, liberales, optimistas, abiertos al cambio, siempre conectados, inmersos en la tecnología digital y las redes sociales, esto les ha permitido centrar su búsqueda en recomendaciones de personas de su círculo social, amigos y personajes referentes que aparecen en diversas plataformas digitales (Castro et al., 2018). Los millennials continúan incorporándose al campo laboral, y su poder adquisitivo aumenta, esto representa un posible mercado significativo para 
la industria turística (Manfreda, Ljubi y Groznik, 2019; Shamala, 2013). El turismo que realizan los jóvenes representa un nicho de mercado importante y atractivo para la industria turística, producto de un comportamiento heterogéneo que para las generaciones mayores de 40 años podría entenderse como liberal (Posso y Cardona, 2016).

En los jóvenes millennials, sus comportamientos de viaje son más sostenibles que otras generaciones, experimentan disminución general en el uso del automóvil, tienen hábitos menos contaminantes (Manfreda et al., 2019). A pesar de que compran mediante Internet, es algo habitual para la generación de los millennials revisan con mucho cuidado el proceso de pago en las plataformas antes de asegurar la transacción, este comportamiento de desconfianza es por la información obtenida de otros usuarios los cuales han compartido su experiencia sobre este proceso (Valdez-Roca, 2018). Otra característica de los turistas de la generación millennials, es que son flexibles al momento de viajar en cuanto a la fecha y el destino, su patrón de consumo no es fácil de predecir, no tiene una preferencia específica, sin embargo, la influencia recibida puede derivarse por las vivencias revisadas en los canales de personas influyentes en Internet, además se inclinan por asegurar tiempo para la diversión durante su estancia en el destino (Carvalho dos Santos et al., 2015). Por lo tanto, las empresas turísticas, deben contemplar diferentes tácticas y estrategias dinámicas para ganarse su lealtad y tratar de comprender su comportamiento, siendo que las generaciones que vienen detrás de ellos pueden ser más difíciles de predecir considerando las tendencias actuales.

La reputación de la empresa como marca en Internet, es un componente fundamental que condiciona la decisión de compra de los nativos digitales de la generación Y, el mantenimiento de la estrategia de gestión de relaciones con los clientes en redes sociales se convierte en un medio necesario para las empresas turísticas en la comunicación con los consumidores (Prasad, Garg y Prasad, 2019). Es importante que la organización, esté activa atendiendo las necesidades y expectativas de este segmento, los millennials son menos leales a las compañías de viajes y hospedaje, están dispuestos a cambiar de aerolínea u hotel si consideran que el programa de fidelización ofrece un mejor producto en su conjunto (Lee, 2014). La 
también llamada generación Y, está altamente influenciada por los desarrollos tecnológicos, es el primer segmento de población con un estilo de vida basado en la digitalización de los servicios, además, las redes sociales se han vuelto una herramienta eficaz para buscar, compartir, colaborar y consumir todo tipo de información utilizada en la toma de decisiones (Alan y Kabadayı, 2016; Liberato et al., 2018). Esta generación son expertos en el uso de dispositivos digitales (Lee y Engelman, 2012), por consiguiente, resulta de interés conocer los factores que, les conducen a seleccionar los lugares que cumplen con las expectativas indispensables, para solventar las necesidades básicas durante su estancia en el destino al cumplir una agenda empresarial o por vacaciones.

\section{METODOLOGÍA}

Para el presente trabajo, se optó por atraer como población de estudio a las personas de la generación millennials nacidas entre 1981 hasta el año 2000 (Carvalho dos Santos et al., 2015), residentes de la ciudad de Tijuana, Baja California, los cuales realizaron al menos cinco viajes fuera del Estado de Baja California en 2019. La población de este municipio es aproximadamente de 1 millón 773 mil 558 habitantes, de los cuales 591.336 tienen entre 20 y 39 años en correspondencia al rango de este segmento de la población (Comité Estatal de Planeación para el Desarrollo (COPLADE), 2017). Estos datos fueron la base para adquirir la muestra representativa de 385 individuos a los que se les aplicó el instrumento, mediante el uso de la fórmula aplicada al tamaño de la población, con 95\% de confianza y 5\% de error admitido (Hernández, Fernández, y Baptista, 2014). Se analizó los datos obtenidos mediante el cuestionario con seis dimensiones (Tabla 1) y con el propósito de describir y correlacionar las variables analizadas.

Tabla 1. Dimensiones del cuestionario aplicado.

\begin{tabular}{|c|c|c|}
\hline Dimensiones & $\begin{array}{c}\text { Número de } \\
\text { ítems }\end{array}$ & $\begin{array}{c}\text { Tipo de } \\
\text { preguntas }\end{array}$ \\
\hline $\begin{array}{c}\text { Entorno empleado para reservar el servicio de avión; medio que utiliza } \\
\text { para informarse del destino; donde se hospedó. }\end{array}$ & 5 & Opción múltiple \\
\hline Servicios tecnológicos utilizados durante su viaje. & 14 & Escala de Likert \\
\hline Medio que utiliza con mayor frecuencia para realizar recomendaciones. & 1 & Opción múltiple \\
\hline
\end{tabular}




\begin{tabular}{|c|c|c|}
\hline Dimensiones & $\begin{array}{c}\text { Número de } \\
\text { ítems }\end{array}$ & $\begin{array}{c}\text { Tipo de } \\
\text { preguntas }\end{array}$ \\
\hline Frecuencia con que utiliza las apps de Internet. & 15 & Escala de Likert \\
\hline Contribución de los servicios digitales en la experiencia de viaje. & 9 & Escala de Likert \\
\hline Información socioeconómica de la persona encuestada. & 3 & Opción múltiple \\
\hline
\end{tabular}

Fuente: elaboración propia.

En el análisis estadístico de confiabilidad con alfa de Cronbach utilizando el programa IBM SPSS Statistics 20, para este proceso se incluyeron las preguntas descritas en las dimensiones del cuestionario con escala de Likert, al obtener valores por encima de 0,7 se tiene consistencia interna (Hinton, Mcmuray y Browlow, 2014) lo que se traduce en buena confiabilidad para el instrumento aplicado a la población asumida en la investigación (Tabla 2).

Tabla 2. Resultados de estadísticas de confiabilidad con alfa de Cronbach.

\begin{tabular}{|c|c|c|c|c|c|}
\hline $\begin{array}{c}\text { Servicios tecnológicos } \\
\text { utilizados durante su viaje }\end{array}$ & \multicolumn{2}{|c|}{$\begin{array}{c}\text { Frecuencia con que utiliza } \\
\text { las apps de Internet }\end{array}$} & \multicolumn{2}{|c|}{$\begin{array}{c}\text { Contribución de los } \\
\text { servicios digitales en la } \\
\text { experiencia de viaje }\end{array}$} \\
\hline $\begin{array}{c}\text { Alfa de } \\
\text { Cronbach }\end{array}$ & $\begin{array}{c}\mathrm{N} \text { de } \\
\text { elementos }\end{array}$ & $\begin{array}{c}\text { Alfa de } \\
\text { Cronbach }\end{array}$ & $\begin{array}{c}\mathrm{N} \text { de } \\
\text { elementos }\end{array}$ & $\begin{array}{c}\text { Alfa de } \\
\text { Cronbach }\end{array}$ & $\begin{array}{c}\mathrm{N} \mathrm{de} \\
\text { elementos }\end{array}$ \\
\hline 0,928 & 13 & 0,839 & 16 & 0,953 & 9 \\
\hline
\end{tabular}

Fuente: elaboración propia.

Se inició por la aplicación de la estadística descriptiva con la finalidad de revisar las tendencias en el uso de los servicios digitales. Posterior el análisis de regresión lineal múltiple, para identificar la relación que tiene la utilización de servicios tecnológicos y apps de Internet empleadas durante el viaje con la experiencia obtenida.

\section{RESULTADOS}

Los resultados obtenidos, en cuanto al lugar donde se hospedaron en su último viaje, los tres primeros en ese orden son: alojarse en hotel, seguido de llegar con familiares o amigos y rentaron departamento (Figura 
1). Cabe destacar que, los encuestados utilizaron algún medio para reserva del servicio de hospedaje, como hotel $(40,5 \%)$, departamento $(13,8 \%)$, rentar casa $(8,3 \%)$, hostal $(1,6 \%)$ y cabaña $(1,3 \%)$.

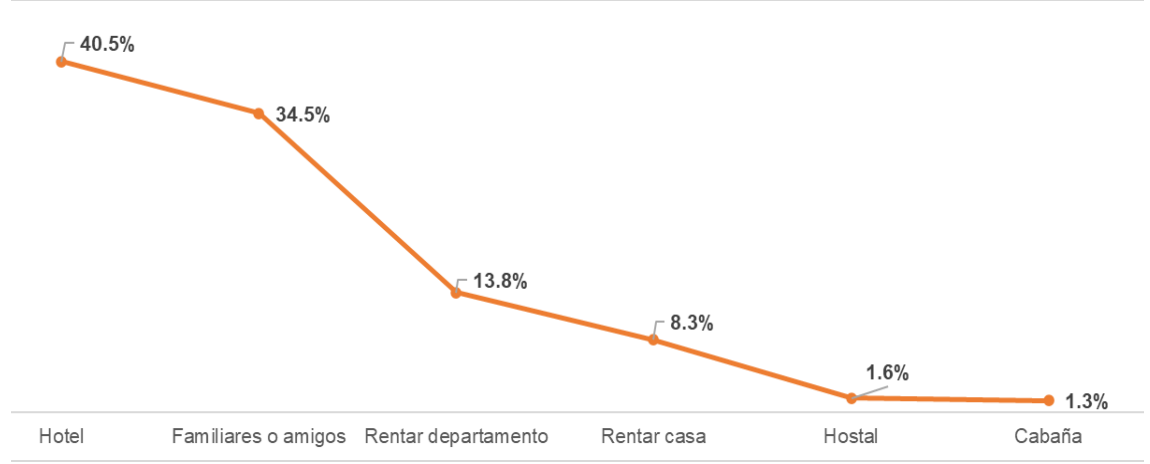

Figura 1. Lugar de hospedaje.

Fuente: elaboración propia.

Para informarse de los servicios y opciones disponibles para los visitantes en el destino, los jóvenes de la generación millennials principalmente buscan mediante el motor de búsqueda de Google con 34,3\%, seguido de preguntar con familiares y amigos $(24,4 \%)$ de las recomendaciones y experiencias obtenidas de los lugares de interés, también averiguan con información disponible en Facebook (11,2\%) (Figura 2).

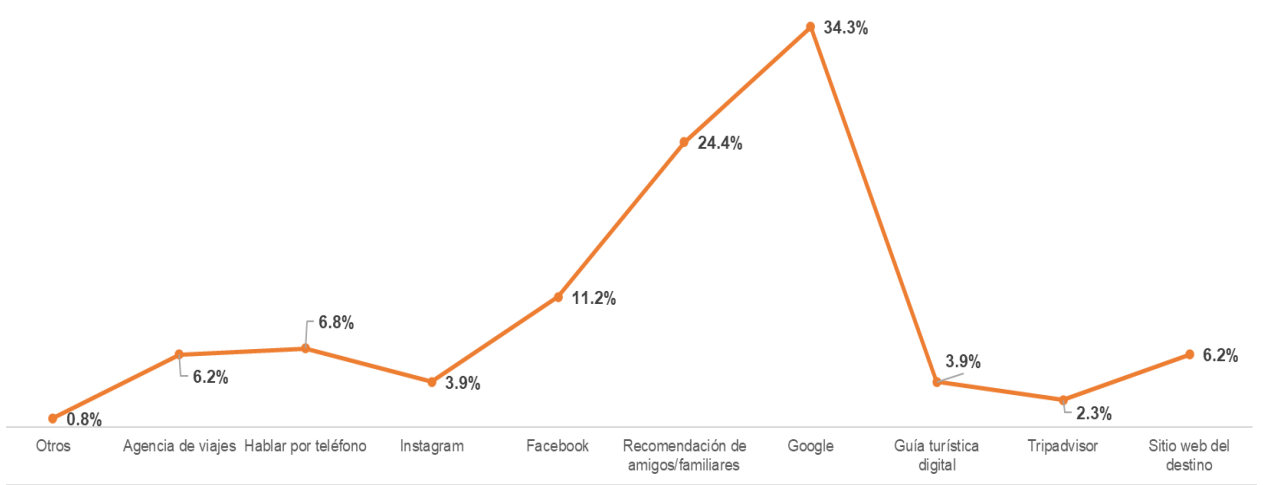

Figura 2. Medio que más utiliza para informarse del destino que visita. Fuente: elaboración propia. 
Las herramientas digitales más utilizadas por los individuos de la generación millennials para compartir experiencias durante el viaje se acentúa con mayor porcentaje en la opción de Facebook $(42,1 \%)$, otra proporción importante prioriza esta actividad mediante Instagram (18,2\%), seguido de Google (14,8\%) que se caracteriza por permitir la aportación de comentarios y calificar los diversos lugares visitados (Figura 3). Asimismo, 17,1\% de los encuestados, omite compartir su experiencia durante el viaje. Las aplicaciones para viajeros entre las que se encuentran Yelp $(1,6 \%)$ y Tripadvisor $(1,3 \%)$, no son las más importantes para este segmento de la población.

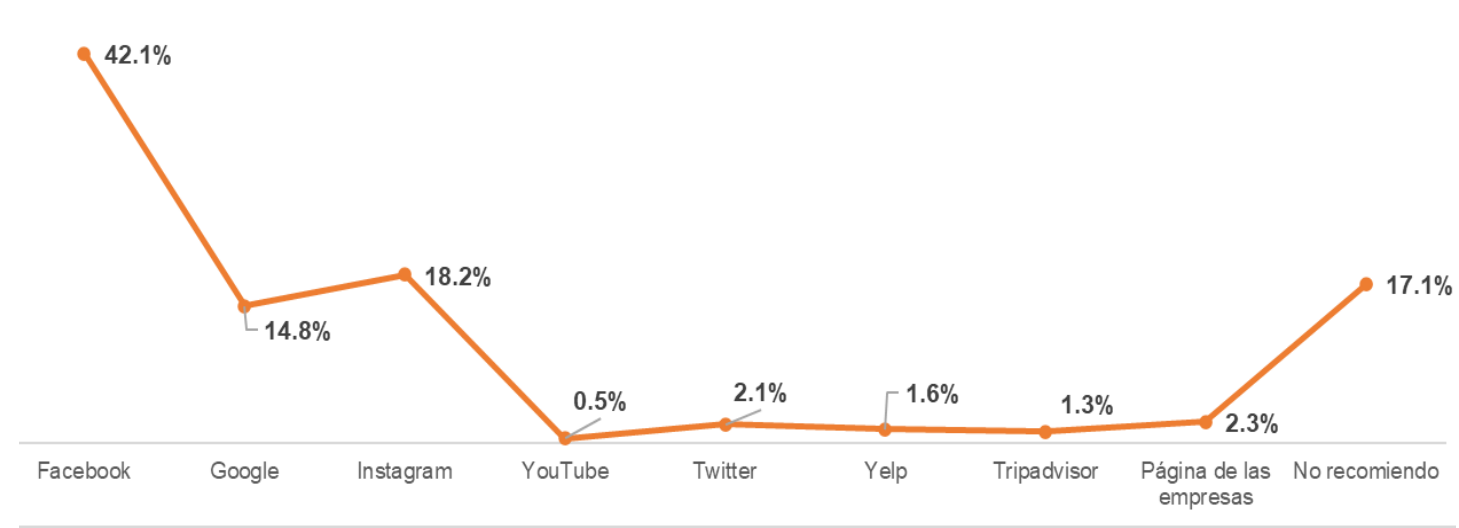

Figura 3. Medio que utiliza para realizar recomendaciones durante su estancia.

Fuente: elaboración propia.

Se preguntó la frecuencia de uso en una escala del 1 al 5 con base en la incidencia en que utilizan los servicios tecnológicos los turistas millennials, en esto se destacan los cinco principales (Figura 4), con mayor importancia se presenta el uso del sistema de geolocalización $(3,88)$, consulta del clima $(3,79)$, búsqueda de restaurantes $(3,69)$, servicio de taxi privado $(3,60)$ por sistema o aplicación de Internet resulta fundamental, por la seguridad de la persona y necesidad de movilidad en la ciudad. Además, se integra compartir experiencias en redes sociales $(3,54)$. 


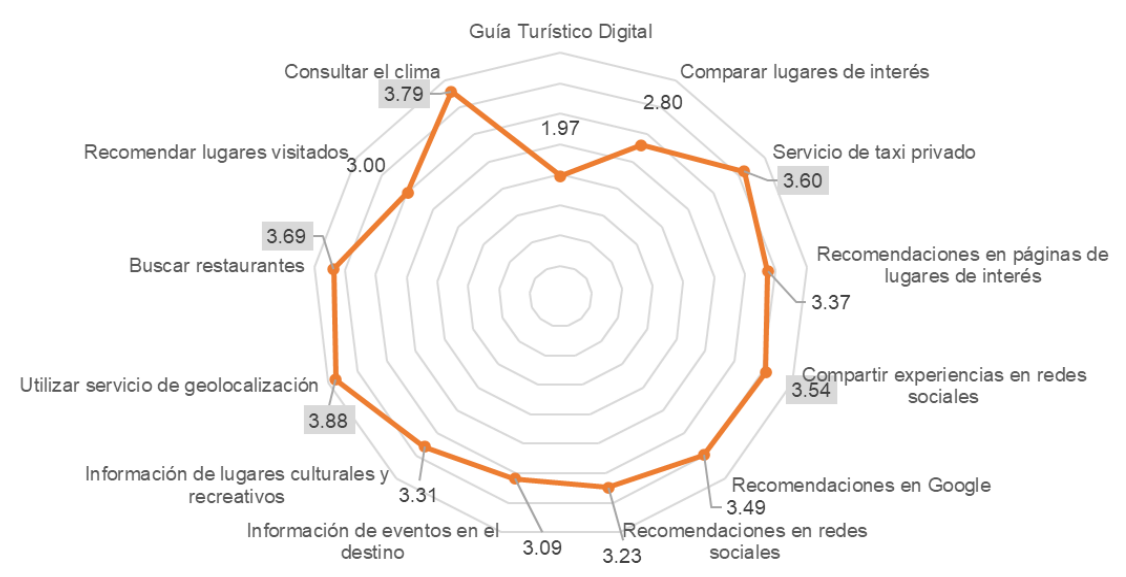

Figura 4. Servicios tecnológicos utilizados durante el viaje.

Fuente: elaboración propia.

Las apps instaladas en el Smartphone empleadas durante el viaje, se preguntó la frecuencia de uso en una escala del 1 al 5, en los resultados se anteponen las redes sociales, Facebook (4,13), WhatsApp (4,07) e Instagram (3.87) (Figura 5). Otras aplicaciones que pueden considerarse con acceso importante son el servicio de taxi privado (Uber, 3.64) y consulta de YouTube $(3,17)$.

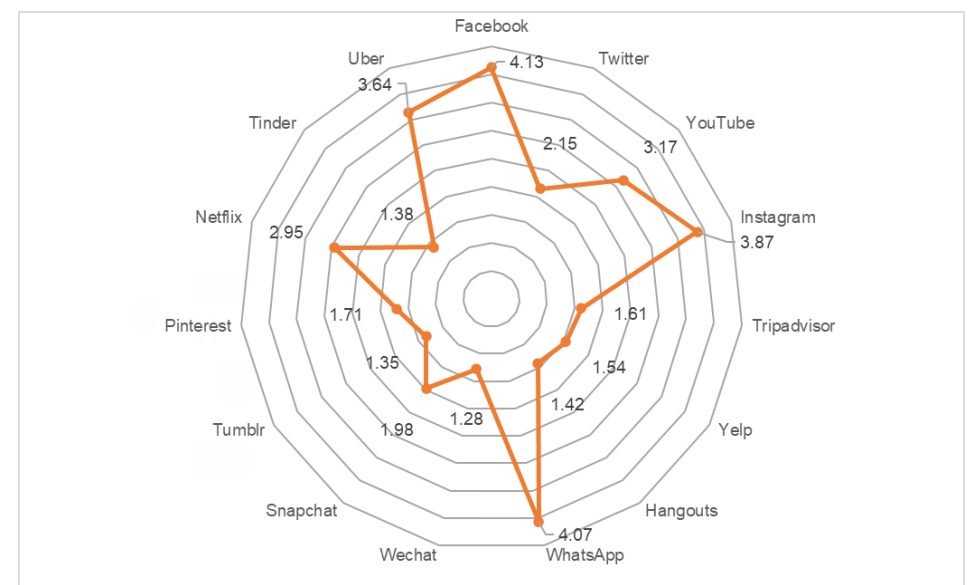

Figura 5. Apps utilizadas durante el viaje.

Fuente: elaboración propia. 
Para conocer el efecto de las tecnologías en la experiencia de los viajeros de la generación millennials, se realizó un análisis de regresión múltiple (Tabla 3). Se encontró una correlación media positiva $(0,590)$, entre la frecuencia con que utilizan los servicios tecnológicos; empleo de las apps instaladas en sus dispositivos móviles inteligentes (variables independientes) durante su visita; con la relación de estos dos elementos en la experiencia de viaje en este segmento de la población (variable dependiente). En este resultado también se añade, el valor de R cuadrado corregida (0,345), que implica la aportación de estas variables, destacándose el total de la varianza que se explica en la variable dependiente producto de las dependientes. Es importante enfatizar, la aparición de la tecnología como un componente clave en la industria turística, que se incorpora a otros elementos ya existentes (Infraestructura, demanda del destino, servicio al cliente, atractivos disponibles, etc.), los cuales se tratan en otros estudios.

Tabla 3. Análisis de regresión múltiple: servicios tecnológicos y apps de Internet utilizados - contribución de los servicios digitales en la experiencia de viaje.

\begin{tabular}{|c|c|c|c|c|}
\hline \multicolumn{5}{|c|}{ Resumen del modelo } \\
\hline Modelo & $\mathbf{R}$ & R cuadrado & $\begin{array}{c}\text { R cuadrado } \\
\text { corregida }\end{array}$ & $\begin{array}{c}\text { Error típ. de la } \\
\text { estimación }\end{array}$ \\
\hline 1 & $0,590 a$ & 0,348 & 0,345 & 0,86737 \\
\hline a. Variables predictoras: (Constante), servicios tecnológicos, apps \\
\hline
\end{tabular}

Fuente: elaboración propia.

Asociado al resultado obtenido en la Tabla 3, mediante la prueba ANOVA (Tabla 4) se obtuvo el coeficiente de significancia de 0,01 entre los elementos estudiados en la prueba estadística en relación a las variables independientes (frecuencia con que utilizan los servicios tecnológicos; empleo de las apps instaladas en sus dispositivos móviles inteligentes); con la variable dependiente (experiencia de viaje a partir del acceso a las tecnologías durante la visita al destino). Por lo tanto, es posible confirmar la validez del análisis de regresión, el modelo lineal es adecuado y significativo en el nivel de 0,01. 
Tabla 4. Significancia: ANOVA.

\begin{tabular}{|c|c|c|c|c|c|c|}
\hline \multicolumn{7}{|c|}{ ANOVA } \\
\hline \multicolumn{2}{|r|}{ Modelo } & $\begin{array}{l}\text { Suma de } \\
\text { cuadrados }\end{array}$ & gl & $\begin{array}{c}\text { Media } \\
\text { cuadrática }\end{array}$ & $\mathbf{F}$ & Sig. \\
\hline \multirow{3}{*}{1} & Regresión & 153.450 & 2 & 76,725 & 101.983 & $0,000 \mathrm{~b}$ \\
\hline & Residual & 287.390 & 382 & 0,752 & & \\
\hline & Total & 440.840 & 384 & & & \\
\hline \multicolumn{7}{|c|}{ a. Variable dependiente: experiencia del viaje } \\
\hline & b. Variak & predictoras: & stant & servicios tecr & ógicos, ap & \\
\hline
\end{tabular}

Fuente: elaboración propia.

\section{CONCLUSIONES}

Los viajeros millennials, reservan su vuelo y alojamiento a través del sitio de Internet de la aerolínea y sitios web del hotel, respectivamente, seguido de apps instaladas en su dispositivo móvil. Asimismo, revisan oferta de alojamientos particulares y turísticos por Airbnb, y en web de servicio de alojamiento (trivago, booking, expedia, kayak, etc.).

Los millennials comparten su experiencia durante el viaje en las redes sociales, como Facebook, Instagram y en el sistema de recomendaciones Google. Para enterarse de los servicios y opciones disponibles en el destino utilizan el motor de búsquedas instalado en Google, también optan por preguntar a sus familiares y amigos de las sus experiencias obtenidas, además revisan la información disponible en la red rocial Facebook. Aunado a lo anterior, las aplicaciones que tienen mayor actividad durante su travesía en el destino coinciden con Facebook e Instagram y se añade la herramienta de mensajería instantánea WhatsApp, está última ha tenido una evolución favorable, hasta ser considerada estar en la categoría de red social. Una de las características documentadas que distingue a los millennials es la flexibilidad en cuando la forma y momento de viajar, su patrón de consumo es complejo de predecir y fidelizar, por lo tanto, es necesario mantener diversos canales de comunicación activos, para el desarrollo de una 
estrategia de entrega de valor en el servicio, con el objetivo de anticiparse a las tendencias que sigue este segmento de la población.

También, es importante destacar la correlación media positiva que tiene la frecuencia con que utilizan los servicios tecnológicos y empleo de las apps con la experiencia de viaje de los millennials, esta asociación se da en un 34,5\%. Esto crea la oportunidad para usar la tecnología en beneficio de la experiencia del turista y la sostenibilidad del destino, mediante el uso de las TIC.

\section{REFERENCIAS BIBLIOGRÁFICAS}

Alan, A. K., y Kabadayı, E. T. (2016). The Effect of Personal Factors on Social Media Usage of Young Consumers. Procedia - Social and Behavioral Sciences, 235, 595-602. https://doi.org/10.1016/j.sbspro.2016.11.086

Ballesteros, B., Tavera, J. F., y Castaño, D. (2014). Aceptación tecnológica de la publicidad en dispositivos móviles en Colombia. Semestre Económico, 17(36), 133-153. https://doi.org/10.22395/ seec.v17n36a6

Caballero, M., y Baigorri, A. (2013). ¿Es operativo el concepto de generación? Aposta. Revista de Ciencias Sociales, 56, 1-45. http://www.redalyc.org/articulo.oa?id=495950252001

Carvalho dos Santos, J., de Oliveira, M., Do Nascimento, B., y Prudêncio, J. (2015). Carrera, renta y consumo bajo la perspectiva del joven de la generación "Y". Invenio, 18(24), 119-135. https://www.redalyc.org/articulo.oa?id=87739279009

Castro, D., Peñaloza, L., y Tamayo, A. (2018). Tecnologías en línea populares para viajar: ¿cuáles utilizan los jóvenes universitarios para hacer turismo? Actualidades Investigativas En Educación, 18(2), https://doi.org/10.15517/aie.v18i2.33149 
Chang, S. E., y Wei-Cheng, S. (2018.). Exploring smartphone social networking services for mobile tourism. International fournal of Mobile Communications, 16(1), 63-81. https://doi.org/10.1504/ IJMG.2018.10007781

Comité Estatal de Planeación para el Desarrollo (COPLADE). (2017). Publicaciones Sociodemográficas: Tijuana, Baja California. http://www.bajacalifornia.gob.mx/portal/nuestro_estado/ municipios/tijuana/tijuana.jsp

Díaz-Sarmiento, G., López-Lambraño, M., y Roncallo-Lafont, L. (2017). Entendiendo las generaciones: una revisión del concepto, clasificación y características distintivas de los Baby Boomers, X Y Millennials. Clío América, 11(22), 188-204. https://dialnet.unirioja.es/servlet/ articulo? codigo $=6509216$

Dickinson, J. E., Hibbert, J. F., y Filimonau, V. (2016). Mobile technology and the tourist experience: (Dis)connection at the campsite. Tourism Management, 57, 193-201. https://doi.org/10.1016/j. tourman.2016.06.005

Djamasbi, S., Siegel, M., y Tullis, T. (2010). Generation Y, web design, and eye tracking. International Journal of Human Computer Studies, 68(5), 307-323. https://doi.org/10.1016/j.ijhcs.2009.12.006

Feixa, C., Fernández-Planells, A., y Figueras-Maz, M. (2016). Generación Hashtag. Los movimientos juveniles en la era de la web social. Revista Latinoamericana En Ciencias Sociales, Niñez y Fuventud, 14(1), 107-120. https://doi.org/10.11600/1692715x.1416301115

Hernández, R., Fernández, G., y Baptista, P. (2014). Metodología de la Investigación. McGraw Hill.

Hinton, P., Mcmuray, I., y Browlow, C. (2014). SPSS Explained. Routledge. 
Lee, D., y Engelman, K. (2012). The impact of mobile on GPG CRM strategy: A Kraft Canada case study. International fournal of Mobile Marketing, 7(1), 5-22. https://www.mmaglobal.com/international_journal_mobile_marketing_canada_vol7_nol

Lee, T. Y. (2014). Segmenting Millennial Travelers Business vs Leisure. HVS. https://www.hvs.com/article/6737-segmenting-millennial-travelers-business-vs-leisure

Liberato, P., Liberato, D., Abreu, A., Alén-González, E., y Rocha, Á. (2018). Generation Y: The Competitiveness of the Tourism Sector Based on Digital Technology. Advances in Intelligent Systems and Computing, 724. https://doi.org/10.1007/978-3-319-74980-8_21

Linares, J., y Vásquez, K. (2018). Ciudades inteligentes: ¿̇materialización de la sostenibilidad o estrategia económica del modelo neoliberal? Agora U.S.B., 18(2), 479-495. https://doi. org/10.21500/16578031.3134

López-Rodríguez, A., y López, S. (2018). Impacto de las TIC en el turismo: Caso colombiano. Cuadernos de Turismo, 41, 399-418. https://doi.org/10.6018/turismo.41.327081

Luo, J., Dey, B. L., Yalkin, C., Sivarajah, U., Punjaisri, K., Huang, Y., y Yen, D. A. (2018). Millennial Chinese consumers' perceived destination brand value. Journal of Business Research, 116. 655-665. https://doi.org/10.1016/j.jbusres.2018.06.015

Manfreda, A., Ljubi, K., y Groznik, A. (2019). Autonomous vehicles in the smart city era: An empirical study of adoption factors important for millennials. International fournal of Information Management. https://doi.org/10.1016/j.ijinfomgt.2019.102050

Melián, S., y Bulchand, J. (2015). Segunda economía en el sector turístico: TIC y puestos de trabajo. Pasos. Revista de Turismo y Patrimonio Cultural. 13(5), 1265-1275. https://www.redalyc. org/pdf/881/88142120018.pdf 
Ordaz-Mejía, D. A., y Osorio-García, M. (2016). Significados del viaje turístico en jóvenes millenials. Caso de estudio: Texcoco, México. Revista Latinoamericana de Ciencias Sociales, Niñez y Juventud, 16(2), 897-912. https://doi.org/10.11600/1692715x.16217

Organización Mundial del Turismo. (2015). Las tecnologías al servicio del Turismo para Todos. https:// www.unwto.org/es/archive/global/event/las-tecnologias-al-servicio-del-turismo-para-todos

Ponsignon, F., y Derbaix, M. (2020). The impact of interactive technologies on the social experience: An empirical study in a cultural tourism context. Tourism Management Perspectives, 35. https://doi. org/10.1016/j.tmp.2020.100723

Posso, Á., y Gardona, D. (2016). Tendencias de consumo turístico de los Millennials en la ciudad de Ibarra. Ecos de la Academia, 4, 145-157. http://revistasojs.utn.edu.ec/index.php/ecosaca$\mathrm{demia/article/view/170/166}$

Prasad, S., Garg, A., y Prasad, S. (2019). Purchase decision of generation Y in an on-line environment. Marketing Intelligence and Planning, 37(4), 372-385. https://doi.org/10.1108/MIP-022018-0070

Ricaurte-Quijano, G., Arellano, A., y Naranjo, K. (2017). (Des)conexión durante el viaje turístico: uso de Smartphone por parte de viajeros independientes en la ciudad de Guayaquil. Teoría y Praxis, 23, 93-119. https://doi.org/10.22403/UQROOMX/TYP23/04

Rodríguez-Moreno, D. (2018). Tecnologías de información y comunicación para el turismo inclusivo. Revista Facultad de Ciencias Económicas, 26(1). https://doi.org/10.18359/rfce.3142

Rossi, L., Maris, S., Garzaniti, R., Biganzoli, B., y Llanos, G. (2018). La inteligencia a través de las generaciones: Millennials y centennials. Acta de Investigación Psicológica, 8(2), 90-100. https:/ / doi. org/10.22201/fpsi.20074719e.2018.2.08 
Secretaría de Turismo (SEGTUR). (2018). Visión Global del Turismo a México. Ciudad de México. https://www.datatur.sectur.gob.mx/Documentos\%20compartidos/VisionGlobalTurismoAMexAbr2018.pdf

Shamala, D.N. (2013). Consumo Global de Medios Móviles: Llegar a los Millennials. https://www.inmobi. com/blog/2013/02/26/Global-Mobile-Media-Consumption-Reaching-Millennials

Simanjuntak, M. (2019). Generation Y's Complaint Behavior toward On-line Shopping. Independent Fournal of Management and Production, 10(1), 101. https://doi.org/10.14807/ijmp.v10i1.839

Valdez-Roca, A. (2018). Un mercado potencial: Perfil turístico de los estudiantes de administración en turismo y hotelería de la Universidad Cesar Vallejo (Lima - Perú). Estudios y Perspectivas En Turismo, 27(3), 756-782. https://www.redalyc.org/articulo.oa?id=180757123020

Vieira, A., Mendes-Filho, L., y do Nascimento, A. (2017). Evaluación de la información de una Aplicación Turística. Estudios y Perspectivas En Turismo, 26(4), 884-904. https://www.redalyc.org/ articulo.oa? $\mathrm{id}=180752919007$

Zhang, T., Gheung, G., y Law, R. (2018). Functionality Evaluation for Destination Marketing Websites in Smart Tourism Cities. Fournal of China Tourism Research, 14(3), 263-278. https://doi.org/1 $0.1080 / 19388160.2018 .1488641$

Zhang, T., Wei, W., Fu, X., Hua, N., y Wang, Y. (2019). Exploring the roles of technology, people, and organization in building a tourism destination experience: Insights from the 2nd USA-China tourism research summit and Industry Dialogue. Journal of Destination Marketing and Management, 12, 130-135. https://doi.org/10.1016/j.jdmm.2019.03.001 
